\title{
Turbulent dispersion properties from a model simulation of the western Mediterranean Sea
}

\author{
H. Nefzi ${ }^{1}$, D. Elhmaidi ${ }^{1}$, and X. Carton ${ }^{2}$ \\ ${ }^{1}$ Faculté des Sciences de Tunis, Université Tunis ElManar, Tunisia \\ ${ }^{2}$ Laboratoire de Physique des Océans-Université Occidentale de Bretagne Brest, France
}

Correspondence to: D. Elhmaidi (dalila.elhmaidi@fst.rnu.tn)

Received: 7 June 2013 - Published in Ocean Sci. Discuss.: 10 July 2013

Revised: 30 January 2014 - Accepted: 4 February 2014 - Published: 14 March 2014

\begin{abstract}
Using a high-resolution primitive equation model of the western Mediterranean Sea, we analyzed the dispersion properties of a set of homogeneously distributed, passive particle pairs. These particles were initially separated by different distances $D_{0}\left(D_{0}=5.55,11.1\right.$ and $\left.16.65 \mathrm{~km}\right)$, and were seeded in the model at initial depths of 44 and $500 \mathrm{~m}$.

This realistic ocean model, which reproduces the main features of the regional circulation, puts into evidence the three well-known regimes of relative dispersion.

The first regime due to the chaotic advection at small scales lasts only a few days ( 3 days at $44 \mathrm{~m}$ depth, a duration comparable with the integral timescale), and the relative dispersion is then exponential. In the second regime, extending from 3 to 20 days, the relative dispersion has a power law $t^{\alpha}$ where $\alpha$ tends to 3 as $D_{0}$ becomes small. In the third regime, a linear growth of the relative dispersion is observed starting from the twentieth day. For the relative diffusivity, the $D^{2}$ growth is followed by the Richardson regime $D^{4 / 3}$. At large scales, where particle velocities are decorrelated, the relative diffusivity is constant.

At $500 \mathrm{~m}$ depth, the integral timescale increases ( $>4$ days) and the intermediate regime becomes narrower than that at $44 \mathrm{~m}$ depth due to the weaker effect of vortices (this effect decreases with depth). The turbulent properties become less intermittent and more homogeneous and the Richardson law takes place.
\end{abstract}

\section{Introduction}

Satellite observations and numerical models of the western Mediterranean Basin have revealed the presence of coherent vortices with different scales (between ten and two hundred kilometers). They are generated by the instability of the inflowing Atlantic water through the Gibraltar strait. Such vortices can survive for long durations (three years), carrying waters characteristic of their formation site and playing an essential role in transport and mixing processes (Millot, 1999; Millot and Taupier-Letage, 2005). In the ocean, mesoscale horizontal velocities are much larger than vertical velocities and the motion is quasi-two-dimensional (McWilliams, 1984).

Numerical simulations of two-dimensional turbulence have shown that the presence of vortices induces an anomalous intermediate dispersion regime between the ballistic regime and the Brownian one. The study of the absolute dispersion of neutral floats in two-dimensional flows and in the western Mediterranean Basin has revealed that the absolute dispersion anomalous regime is characterized by a power law $t^{r}$ where $r$ varies between 5/3 and 5/4 (Elhmaidi et al., 1993, 2010). The corresponding intermediate regime for the relative dispersion is known as the Richardson regime (Richardson, 1926; Babiano et al., 1990).

Many analyses of oceanic data have shown the existence of the Richardson regime. LaCasce and Ohlmann (2003) have used the data from the SCULP (Surface Current and Lagrangian Drift Program) experiment to study the relative dispersion in the Gulf of Mexico. They found an exponential growth in a range of scales from 5 to $50 \mathrm{~km}$ with a duration of about 10 days. At longer times, the time dependence 
was a power law with an exponent lying between 2 and 3 . Ollitrault et al. (2005) analyzed subsurface float pairs in the western and eastern North Atlantic from the TOPOGULF experiment. They found an exponential regime for scales smaller than the Rossby radius of deformation $(D<25 \mathrm{~km})$ and for times $t<6$ days, followed by a Richardson's law from scales 40 to $300 \mathrm{~km}$, and a linear regime was then observed. Lumpkin and Elipot (2010) analyzed the relative dispersion of a surface pair in CLIMODE (the CLIVAR Mode Water Dynamic Experiment) in the Gulf Stream region. They showed the existence of a Richardson regime at scales from $1-3 \mathrm{~km}$ to $300-500 \mathrm{~km}$. At larger scales, the spreading of particles followed a random walk described by a constant diffusivity. The small-scale exponential regime was not observed.

Nevertheless, though large, the number of float or drifter data in the world ocean remains small compared to the ocean surface (or volume), so that their average separation is relatively large and that they do not provide uniform coverage of the ocean. The use of synthetic drifters in numerical simulations can palliate this deficiency and help test the accordance between numerical and experimental data.

Often, particle tracking in numerical models was used to follow the path or mixing of water masses. Here we aim to put into evidence the theoretical regimes of turbulent dispersion in the oceanographic context using a numerical ocean model and particle tracking.

In the present work, we study the dispersion of passive particle pairs, near the surface, in the western Mediterranean Sea. We use a high-resolution model able to reproduce a realistic level of turbulence (mesoscale eddies). In Sect. 2, we recall the numerical conditions of the study (the model and its parameters). In Sect. 3, we briefly recall the theoretical results of turbulent dispersion in quasi-2-D flows. Section 4 is devoted to the analysis of the model results and to the calculation of dispersion and of relative diffusivity. Finally conclusions are drawn.

\section{Material and methods: the ROMS model and particle tracking}

The Regional Ocean Modeling System (ROMS) is a primitive equation model able to simulate both coastal and oceanic dynamics (Shchepetkin and McWilliams, 2005). It is based on the Boussinesq approximation and on the hydrostatic balance. ROMS is a split-explicit, free surface oceanic model discretized in coastline- and terrain-following curvilinear coordinates. Short time steps are used to solve the barotropic momentum equations and longer ones to advance the baroclinic momentum equations. A third-order, upstream-biased advection scheme implemented in ROMS allows the generation of steep gradients, enhancing the effective resolution of the solution for a given grid size (Penven et al., 2008). The explicit lateral viscosity is zero everywhere in the model domain except in the sponge layers near the boundaries where the viscosity increases smoothly toward the lateral open boundaries.

The computational domain for this study extends from $33^{\circ}$ to $44.5^{\circ} \mathrm{N}$ and from $7^{\circ} \mathrm{W}$ to $15^{\circ} \mathrm{E}$. The model grid, forcing, and initial and boundary conditions are built using the ROMSTOOLS package (Shchepetkin and McWilliams, 2005; Penven et al., 2008). The bottom topography is derived from the ETOPO2 $2^{\prime}$ resolution database (Penven and Tan, 2007). A slope parameter $r=2.5$ is used to prevent errors in the computation of the pressure gradient (Smith and Sandwell, 1997). The ROMS configuration used here has a $1 / 8^{\circ}$ horizontal resolution and 20 vertical levels with stretched $s$ coordinates, using surface and bottom stretching parameters $\left(\theta_{\mathrm{s}}=0, \theta_{\mathrm{b}}=6\right)$ (Haidvogel and al., 2000). The temporal resolution is $15 \mathrm{~min}$. Mean monthly temperature and salinity data are obtained from the World Ocean Atlas (WOA2005) database (monthly climatology at $1^{\circ} \times 1^{\circ}$ resolution) (Conkright et al., 2001; Song and Haidvogel, 1994); these data are used to initialize the model and restore conditions in the sponge layers.

Figure 1 represents the model grid and its bathymetry. At the two open boundaries (west and east), an active, implicit, upstream-biased radiation condition connects the model solution to the surrounding sea or ocean (Marchesiello et al., 2001). The model is started from rest and is forced with winds, heat and salinity fluxes from the Comprehensive Ocean-Atmosphere Data Set (COADS05), which is a monthly climatology giving data with a spatial resolution of $0.5^{\circ}$ (Da Silva et al., 1994). The width of the sponge layers is $150 \mathrm{~km}$ and the maximum viscosity in these layers is set to $1000 \mathrm{~m}^{2} \mathrm{~s}^{-1}$.

The ROMS model is integrated until a statistical equilibrium is obtained. Figure 2 a shows the simulated surface currents in a subdomain $\left(2^{\circ} \mathrm{W}\right.$ to $10^{\circ} \mathrm{E}$ and $35.5^{\circ}$ to $\left.40^{\circ} \mathrm{N}\right)$ of the western Mediterranean Basin after the stationary state has been reached. The flow is dominated by coherent vortices with different scales that propagate to the east in accordance with available in situ and satellite data sets (Beranger et al., 2005). A vertical cross section of temperature, salinity and perpendicular horizontal velocity (respectively Fig. 2b, $\mathrm{c}$, and d) through vortex $\mathrm{V}_{1}$ indicates that the thermohaline anomalies associated with this vortex are essentially intensified in the surface layer $(0-200 \mathrm{~m})$, while the dynamical signal extends further down (in particular down to $500 \mathrm{~m}$ depth), though it is weaker at depth than near the surface.

The spatial distribution of the relative vorticity $\varsigma=\frac{\partial v}{\partial x}-$ $\frac{\partial u}{\partial y}$ where $(u, v)$ is the horizontal velocity (Fig. 2e) in a subdomain in the western Mediterranean Basin shows that the flow is dominated by cyclonic and anticyclonic vortices surrounded by filaments with small scales. The horizontal kinetic energy spectrum calculated in this subdomain (figure not shown) indicates the presence of the two inertial ranges with the spectral slopes of two-dimensional turbulence $(-5 / 3$ and -3 , respectively) 


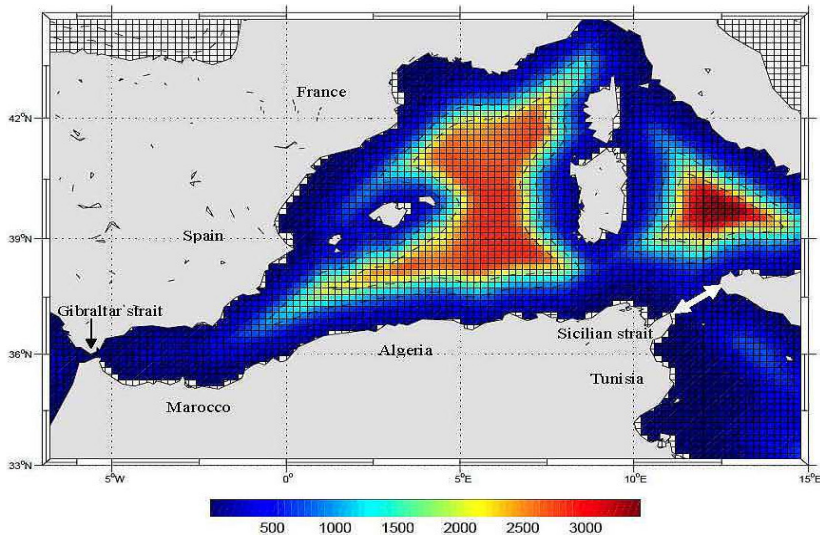

Fig. 1. The model domain and its bathymetry (m).

To study the dispersion processes, we performed two sets of numerical simulations with the ROMS model from the statistical equilibrium state. We seeded neutral, passive particle pairs slightly below the surface (at depth $h=-44 \mathrm{~m}$ ) and at a much larger depth $(h=-500 \mathrm{~m})$. They were advected with the model horizontal velocity. The Lagrangian velocities were calculated using a centered scheme; the interpolation scheme for the particle trajectories was a fourth-order accurate Adams-Bashford-Moulton predictor-corrector scheme. Transport of particles was due to advection only, as no diffusion term was introduced.

\section{Relative dispersion}

To study particle dispersion, relative dispersion and relative diffusivity are commonly used (Richardson, 1926; Babiano et al., 1990). They are defined respectively by

$$
\begin{aligned}
& D^{2}\left(t, D_{0}\right)=\sum_{i \neq j}<x_{i}\left(t, D_{0}\right)-x_{j}\left(t, D_{0}\right)>^{2}, \\
& Y(t)=\frac{1}{2} \frac{\mathrm{d}}{\mathrm{d} t} D^{2}\left(t, D_{0}\right),
\end{aligned}
$$

where $x_{i}\left(t, D_{0}\right)$ and $x_{j}\left(t, D_{0}\right)$ are the vector positions of a pair separated initially by $D_{0}$ and the average is over all particle pairs.

Relative dispersion of particles in a turbulent field is governed by the distribution of velocities with spatial scales. Thus, dispersion is related to the spatial spectrum of kinetic energy. In two-dimensional turbulence, this spectrum has two ranges, called inertial ranges, where nonlinear effects (due to advection) dominate the dynamics. These inertial ranges are, respectively, the range of inverse energy cascade (at larger scales) where the energy cascades upscale, and the range of direct enstrophy cascade (at smaller scales), where the enstrophy cascades downscale. Enstrophy is the square of relative vorticity. In these two ranges, the spectral density of kinetic energy is, respectively,

$$
E(k) \approx k^{-3} \text { and } E(k) \approx k^{-5 / 3},
$$

where $k$ is the horizontal, isotropic wavenumber.

The corresponding Richardson and Lin laws for the relative dispersion of two-dimensional turbulence are a cubic power for the evolution with time in the inverse energy cascade range, and an exponential evolution in the enstrophy cascade range (Babiano et al., 1990).

At intermediate times (in the inverse energy range), the mean square relative velocity defined by $<\delta u^{2}\left(t, D_{0}\right)>=<$ $\frac{\mathrm{d} D}{\mathrm{~d} t} \cdot \frac{\mathrm{d} D}{\mathrm{~d} t}>(D$ is the separation vector of a pair of particles $)$ has linear behavior (Babiano et al., 1990).

After a sufficiently long time, the separation distance of the particle pair reaches the scale of the energetic eddies and the velocities of the two particles decorrelate. Due to homogeneity, the mean-square relative velocity becomes just four times the eddy kinetic energy (Ollitrault et al., 2005; LaCasce and Bower, 2000):

$$
\begin{aligned}
<\left(\frac{\mathrm{d} D}{\mathrm{~d} t}\right)^{2}> & =<\delta u^{2}\left(t, D_{0}\right)> \\
& =<u_{i}^{2}>+<u_{j}^{2}>-2<u_{i} u_{j}>\approx 2<u_{i}^{2}>,
\end{aligned}
$$

where $u_{i}$ and $u_{j}$ are the individual velocities of the particle pair.

The relative diffusivity has two types of variations: $Y(t) \approx$ $D^{2}(t)$ in the enstrophy cascade range, and $Y(t) \approx D^{4 / 3}(t)$ in the inverse energy range.

At larger separation distances, relative dispersion is a random walk and relative diffusivity is constant.

At early times, the individual particle velocities are correlated and the mean-square relative velocity tends toward $Z D_{0}^{2}$, where $Z$ is total enstrophy defined by $Z=\frac{1}{2}<\varsigma^{2}>_{x}$ $\left(<.>_{x}\right.$ is the spatial average of the relative velocity over the entire domain) (Babiano et al., 1990; Ollitrault et al., 2005).

The enstrophy can be calculated through the relative dispersion characteristic time defined by

$\tau(D)=\frac{D^{2}}{Y(t)}$,

which is constant, and tends toward $\frac{2}{Z^{1 / 2}}$ when $D$ tends to zero, and has a power law $\tau(D) \approx D^{2 / 3}$ in the inverse energy cascade range (Babiano et al., 1990).

\section{Results}

2726 passive particles pairs were homogeneously seeded at the subsurface $(h=-44 \mathrm{~m})$ of the model with different separation distances $D_{0}$. Note that the results presented hereafter were independent of this initial depth as long as the particles were seeded in the turbulent surface layer (from 0 to $-100 \mathrm{~m}$ ). The particle initial positions, for the different 

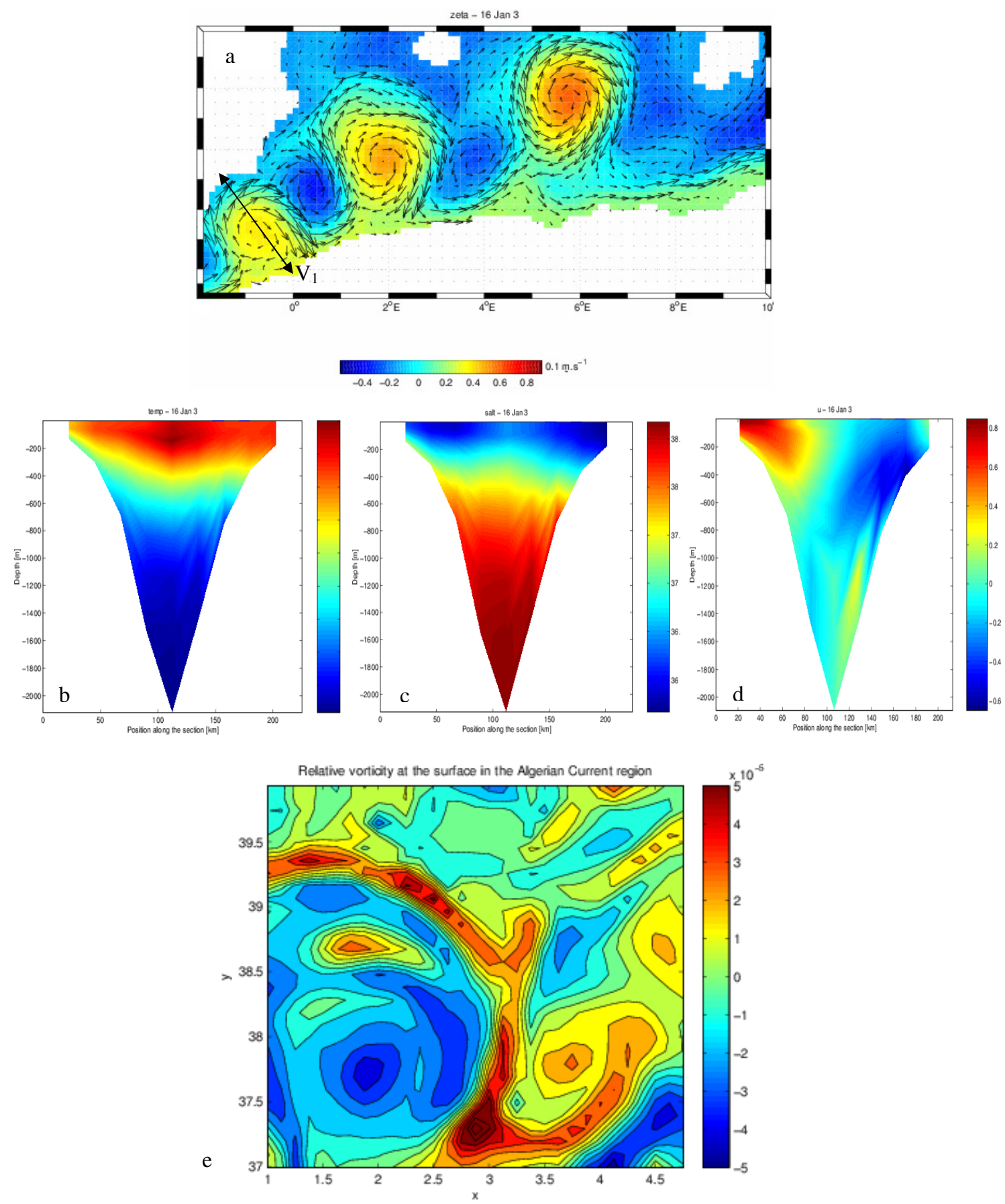

Fig. 2. (a) Simulated surface currents superimposed on the surface elevation in a subdomain of the western Mediterranean Basin. Vertical section of (b) temperature, (c) salinity and (d) perpendicular velocity through vortex $\mathrm{V}_{1}$. (e) Relative vorticity in a subdomain of the western Mediterranean Basin.

simulations, were located between $36^{\circ}$ and $39^{\circ} \mathrm{N}$ in latitude and $0^{\circ}$ and $12^{\circ} \mathrm{E}$ in longitude. They were advected during two months. Then the particles remained at the same depth for the whole duration of the simulations. The float positions, for $D_{0}=5.55 \mathrm{~km}$, are shown in Fig. 3 at the end of the simulation. In the inset we show the initial particle positions.
The particles travel east in accordance with the main circulation. They disperse in the whole domain and some of them aggregate around vortices in energetic domains. This particle evolution is similar to that in two-dimensional turbulence where vortices constitute a barrier transport, trap 


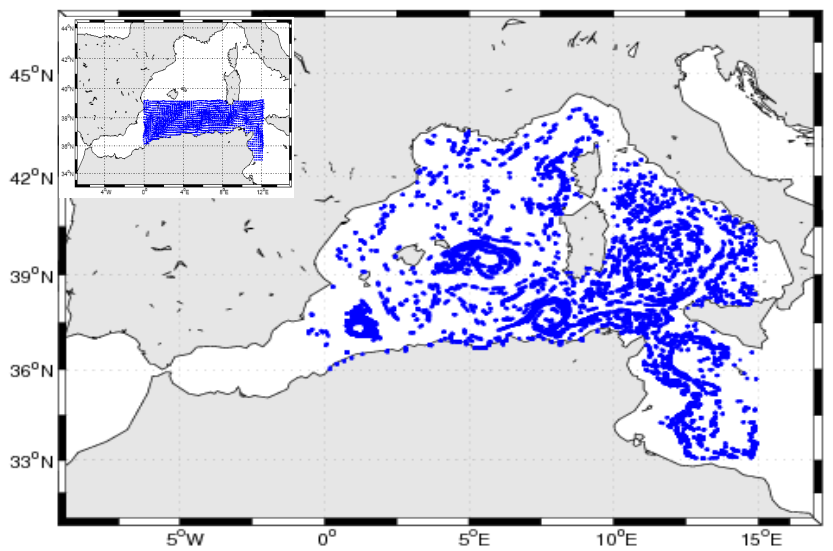

Fig. 3. The particle positions after two months of integration. In the inset we show the initial particle positions.

passive floats and induce a far field effect (Elhmaidi et al., 1993, 2005).

In the western Mediterranean Basin, the zonal motion is dominant. We show in Fig. 4a that the behavior of the total relative dispersion and the zonal one $\left(D_{x}^{2}\left(t, D_{0}\right)=\right.$ $\sum_{i \neq j}<x_{i}\left(t, D_{0}\right)-x_{j}\left(t, D_{0}\right)>^{2}, x_{i}\left(t, D_{0}\right)$ and $x_{j}\left(t, D_{0}\right)$ are the zonal particle positions of a pair separated initially by $\left.D_{0}\right)$ are similar. Due to this similarity, we consider in the following statistics only the zonal components of the particles.

The temporal evolution of the mean square relative velocity $<\delta u^{2}>$ and four times the kinetic energy (4E) are plotted for the value $D_{0}=11.1 \mathrm{~km}$ in Fig. $4 \mathrm{~b}$. This evolution can be described in three different phases. The duration of these phases has been determined by fitting the phenomenological laws and by minimizing the error between the numerical curve and the theoretical laws.

In the first one (lasting during the first three days), the energy remains constant, as the Lagrangian velocities are correlated. This time interval is roughly equal to the Lagrangian integral timescale defined by

$T_{L}=\int_{0}^{\infty} R(\tau) \mathrm{d} \tau$,

where

$R(\tau)=\frac{<u(t) \cdot u(t+\tau)>}{<(u(t) \cdot u(t)>}$

is the Lagrangian velocity autocorrelation function displayed in the inset of Fig. 4b. This Lagrangian integral timescale is about 3 days, as shown by the first root of this function.

In the second phase (lasting from days 3 to 20), the Lagrangian energy initially increases because the particles are trapped in the intense circulation cells around vortices. Then, after day 10 , a decrease in energy can be observed given that the majority of passive particles reach the background field

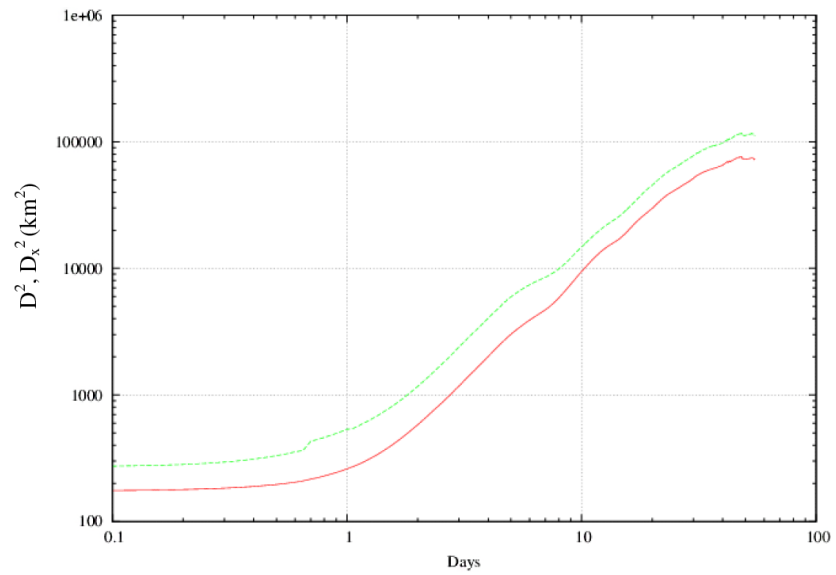

Fig. 4a. Relative dispersion (green curve), zonal relative dispersion (red curve) for $D_{0}=16.65 \mathrm{~km}$.

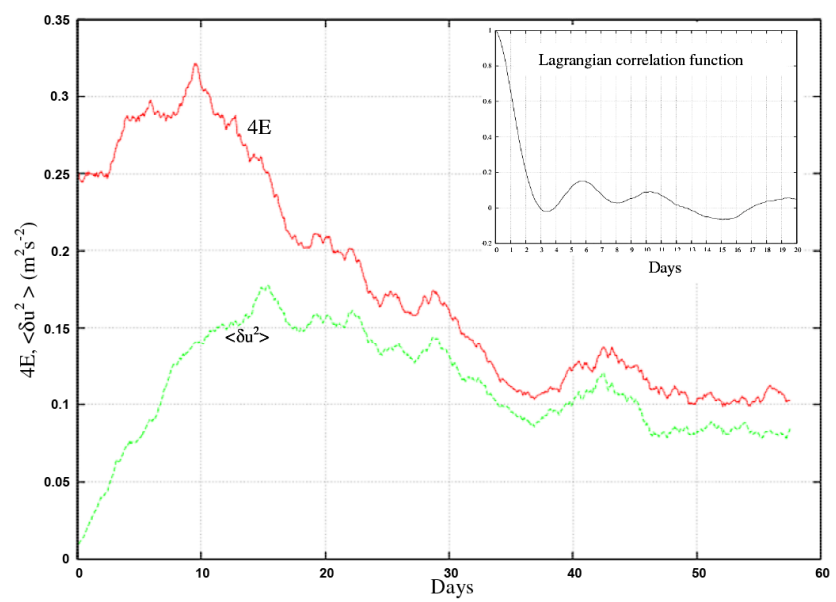

Fig. 4b. Temporal evolution of four times the kinetic energy (4E) (red curve) and of the mean square relative velocity $\left\langle\delta u^{2}\right\rangle$ (green curve) of the particle pairs separated initially by $D_{0}=11.1 \mathrm{~km}$. In the inset we show the temporal evolution of the Lagrangian correlation function at $44 \mathrm{~m}$ depth.

where the energy is low (Elhmaidi et al., 1993). During this phase, the mean square relative velocity has a quasi-linear evolution.

In the final phase $(t>20$ days), the evolutions of $4 \mathrm{E}$ and $<\delta u^{2}>$ are correlated and the mean square relative velocity roughly equilibrates to four times the kinetic energy.

The zonal mean square relative velocity, divided by $t$, is displayed in Fig. 4c for the different separation distances. Between days 3 and $12,<\delta u_{x}^{2}>/ t$ tends to a plateau especially for the smallest value of $D_{0}$. During this time interval, $<\delta u_{x}^{2}>$ has a power law $t^{b}$ where $b$ is roughly equal to 1 , corresponding to the theory (Babiano et al., 1990). For the smallest time and for the different values of $D_{0}=$ $5.55,11.1,16.65 \mathrm{~km}$, we can compute $<\delta u_{x}^{2}>\approx 25,52$, $80 \mathrm{~km}^{2}$ days ${ }^{-2}$, respectively (see the inset of Fig. 4c). As 


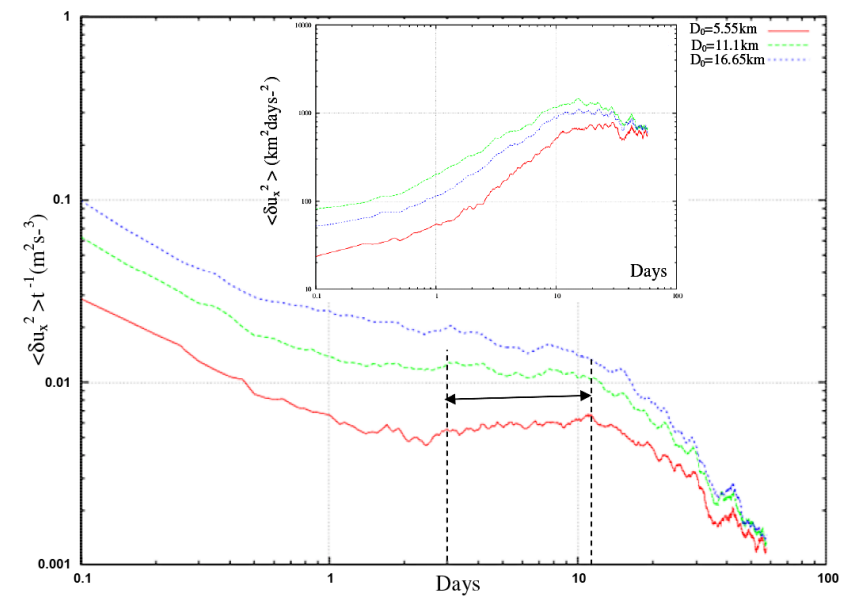

Fig. 4c. Temporal evolution of the mean square relative velocity divided by $t$ for different initial separation distances. The plateau indicates the linear $<\delta u_{x}^{2}>$ law. In the inset, the temporal evolution of $<\delta u_{x}^{2}>$ in $\mathrm{km}^{2}$ days $^{-2}$ is shown.

$<\delta u_{x}^{2}>$ tends to $Z D_{0}^{2}$ for short times, we can deduce that the enstrophy $Z$ fluctuates between 0.3 and 0.81 days $^{-2}$. Using these values of $Z$ and given that $Z=\frac{1}{2}<\varsigma^{2}>_{x}$, the magnitude of the relative vorticity is in accordance with that of Fig. 2e.

In Fig. 5a, the temporal evolution of the zonal relative dispersion is plotted for different separation distances $\left(D_{0}=\right.$ $5.55,11.1$ and $16.6 \mathrm{~km})$. This evolution can be described in three phases.

The first one lasts three days. To analyze the temporal evolution of the zonal relative dispersion in this phase, $D_{x}^{2}$ is displayed in a log-linear plot in Fig. 5b. A linear evolution is observed for the different values of $D_{0}$. This is an indication of the exponential growth, while the dispersion is dominated by eddies with scales larger than the pair separations. Then $D_{x}^{2}$ evolves as $\exp (k t)$ where $k$ varies between 0.74 and 0.82 . This exponential regime was found previously for separation distances smaller than the Rossby radius of deformation (LaCasce and Bower, 2000; LaCasce and Ohlmann, 2003; Ollitrault et al., 2005).

In the second phase $\left(3<t<20\right.$ days), $D_{x}^{2}$ grows like $t^{a}$ where $a$ varies between 1.66 and 2.3. We show in Fig. 5c the temporal evolution of $D_{x}^{2}$ in this range for $D_{0}=16.5 \mathrm{~km}$ and $5.55 \mathrm{~km}$. These two curves are respectively fitted by the linear laws $340 t^{1.66}$ and $13 t^{2.3}$ with a relative error not exceeding $10 \%$.

In the third phase, a linear growth of the relative dispersion is observed starting from the twentieth day.

The zonal relative dispersion coefficient $Y_{x}(D)$ was calculated by finite time differencing $D_{x}^{2}\left(t, D_{0}\right)$. Its evolution versus the distance $D$ is shown in Fig. 6a for several initial distances $D_{0}\left(D_{0}=5.55,11.1\right.$ and $\left.16.6 \mathrm{~km}\right)$. The relative diffusivity is fitted by the curve $0.4 D^{2}$ for $D<20 \mathrm{~km}$, and for $D>20 \mathrm{~km}$ the relative diffusivity is fitted by the curve $4 D^{4 / 3}$

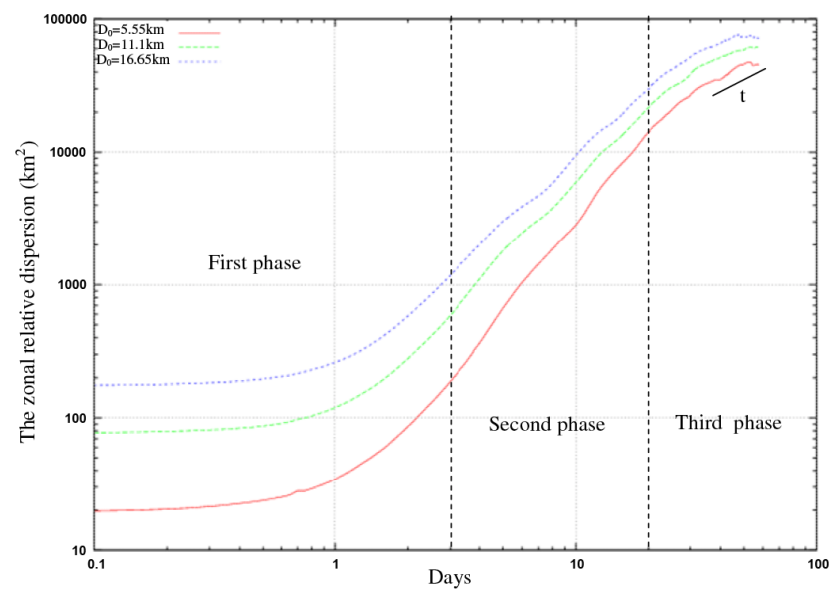

Fig. 5a. Temporal evolution of the zonal relative dispersion $D_{x}^{2}$. The solid black line indicates the linear law.

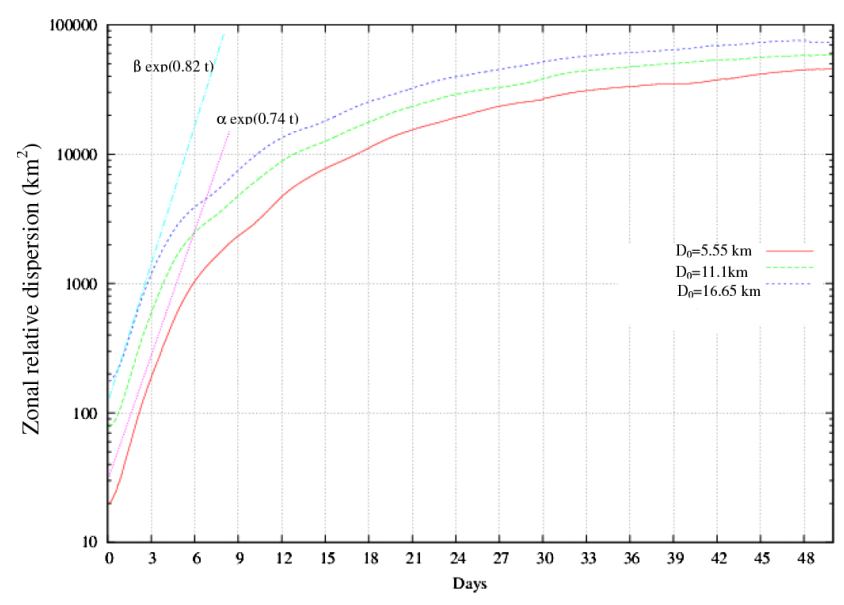

Fig. 5b. The zonal relative dispersion versus time and $D_{0}$. The $\alpha \exp (0.74 t)$ and $\beta \exp (0.82 t)$ laws are given with $\alpha=30$ and $\beta=$ 120.

and $Y(D)$ is constant for distances larger than $100 \mathrm{~km}$. The $D^{2}$ regime confirms the presence of an enstrophy cascade range only for small scales. For large scales, the Richardson $D^{4 / 3}$ is the signature of the inverse energy cascade.

The enstrophy $Z$ of the flow can be calculated via the evolution of the zonal relative characteristic dispersion time $\tau_{x}(D)\left(\tau_{x}(D)=D_{x}^{2} / Y_{x}\right)$ reported in Fig. 6b. For small $D$, the minimum characteristic time varies between two and three days. Then we deduce that the enstrophy fluctuates between 0.44 and $1 \mathrm{day}^{-2}$. This range of enstrophy values is in agreement with that calculated with the minimum of the square relative velocity. For $D>20 \mathrm{~km}, \tau_{x}(D)$ is fitted by the curve $0.3 D^{2 / 3}$ showing the presence of the Richardson regime in the inverse energy cascade range.

In a previous work we have shown that, at large depths, the intermediate absolute dispersion follows a power law over a period of time shorter than near the surface due to the weaker 


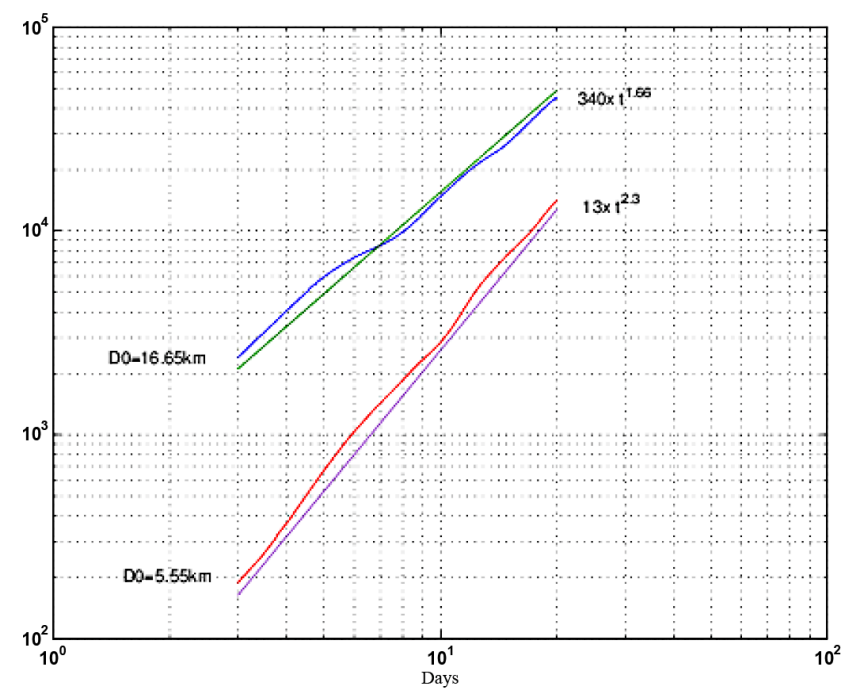

Fig. 5c. Zonal relative dispersion $D_{x}^{2}$ in the second phase ( $3<t<20$ days) for $D_{0}=5.55 \mathrm{~km}$ (red curve) and $D_{0}=16.65 \mathrm{~km}$ (blue curve). The two curves are fitted respectively by $13 t^{2.3}$ (purple curve) and $340 t^{1.66}$ (green curve).

effect of vortices (this effect decreases with depth, Elhmaidi et al., 2010).

To verify this result for the relative dispersion, 833 pairs of particles were seeded at the $500 \mathrm{~m}$ depth initially separated by the values of $D_{0}$ already used. These pairs were released in a rectangular area $\left(37.5^{\circ}\right.$ to $39.1^{\circ} \mathrm{N}$ in latitude and $2^{\circ}$ to $5^{\circ} \mathrm{E}$ in longitude).

The temporal evolution of the surface and deep Lagrangian correlation functions for $D_{0}=5.55 \mathrm{~km}$ (Fig. 7) shows that the surface particle velocities decorrelate more rapidly than the deep one. Thus, the duration of the depth first phase is longer than the surface one. We show in Fig. 8 the surface and the $500 \mathrm{~m}$ depth relative zonal dispersion divided by $t^{3}$ for the smallest value of $D_{0}$. A plateau is well observed for the $500 \mathrm{~m}$ depth relative dispersion from the 6th to the 16th day, which is the signature of the Richardson $t^{3}$ law.

Given the weaker effect of vortices at depth, the turbulent properties become less intermittent and more homogenous and the Richardson law takes place.

Our results are consistent with the analysis of the subsurface drifters released respectively in the Gulf Stream and in the North Atlantic Current at $700 \mathrm{~m}$ depth (LaCasce and Ohlmann, 2003; Ollitrault et al., 2005; Lumpkin, and Elipot, 2010).

\section{Conclusions}

In this work, we have used a high-resolution primitive equation model to study the relative dispersion of neutral passive particle pairs, separated by different initial distances $D_{0}$ $\left(D_{0}=5.55,11.1\right.$ and $\left.16.65 \mathrm{~km}\right)$, deployed in the western

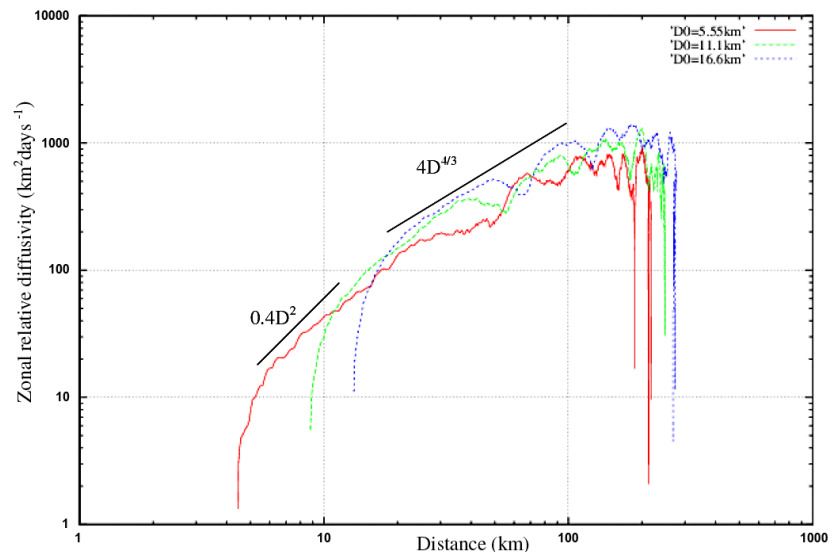

Figure 6a: The zonal relative diffusivity as function of distance D. The $0.4 D^{2}$ and the $4 D^{4 / 3}$ are plotted.

Fig. 6a. The zonal relative diffusivity as a function of distance $D$. $0.4 D^{2}$ and $4 D^{4 / 3}$ are plotted.

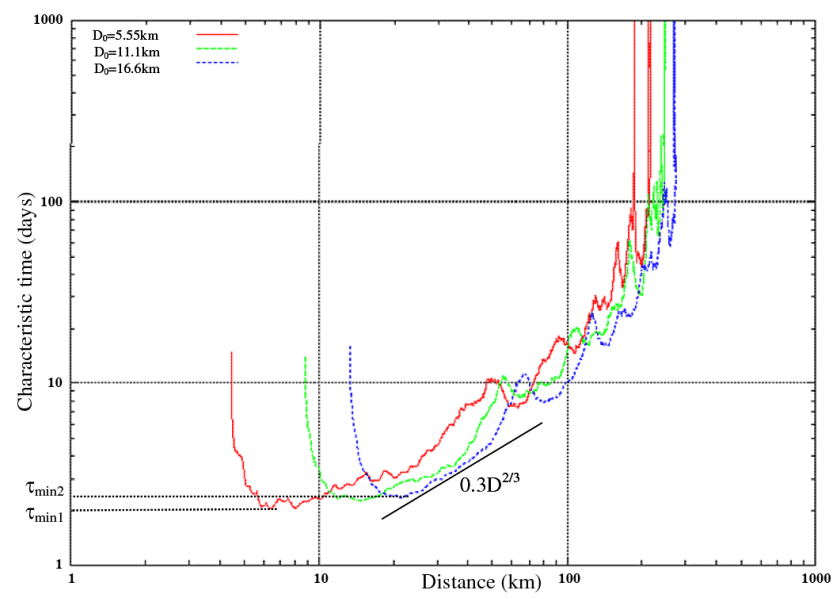

Fig. 6b. The zonal characteristic dispersion time $\tau_{x}(D)=D_{x}^{2} / Y_{x}$ as a function of $D$ and $D_{0} \cdot \tau_{\min 1}=2$ days for $D_{0}=5.55 \mathrm{~km}$ and $\tau_{\min 2}=3$ days. The enstrophy varies between 0.44 and 1 days $^{-2}$. The $0.3 D^{2 / 3}$ curve is given.

Mediterranean Sea at initial depths 44 and $500 \mathrm{~m}$. The particles were advected with the ROMS horizontal model velocity interpolated over the particle trajectories.

The analysis of the model output has shown that the relative dispersion laws are similar to those of 2-D turbulence with the presence of the three well-known phases.

The first phase characterizes the chaotic advection at small scales, lasts only a few days ( 3 days, a duration equivalent to the integral timescale), and the relative dispersion is exponential.

In the second phase, extending from 3 to 20 days, the relative dispersion follows a power law $t^{\alpha}$ where $\alpha$ tends to 3 for small $D_{0}$, according to the Richardson regime.

In the third phase, a linear growth of the relative dispersion is observed starting from the twentieth day. 


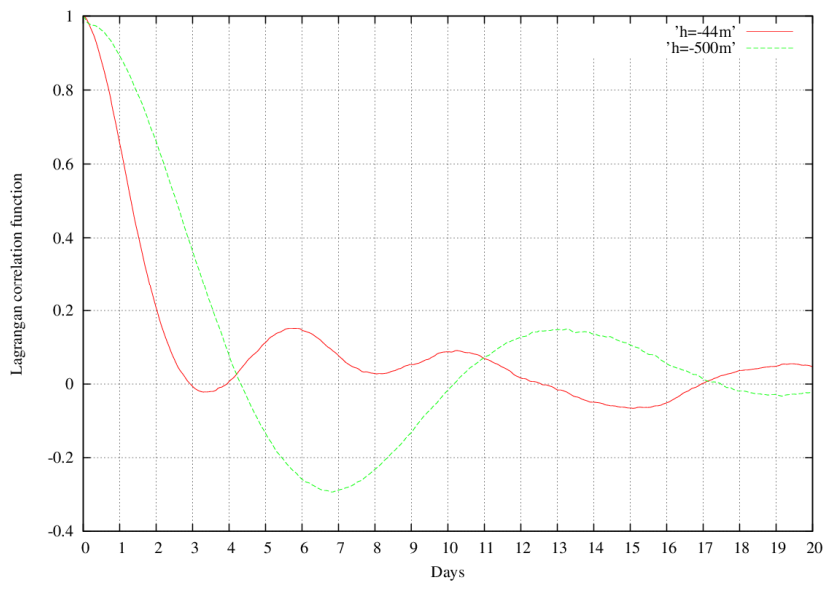

Fig. 7. Temporal evolution of the Lagrangian correlation function at depths 44 and $500 \mathrm{~m}$.

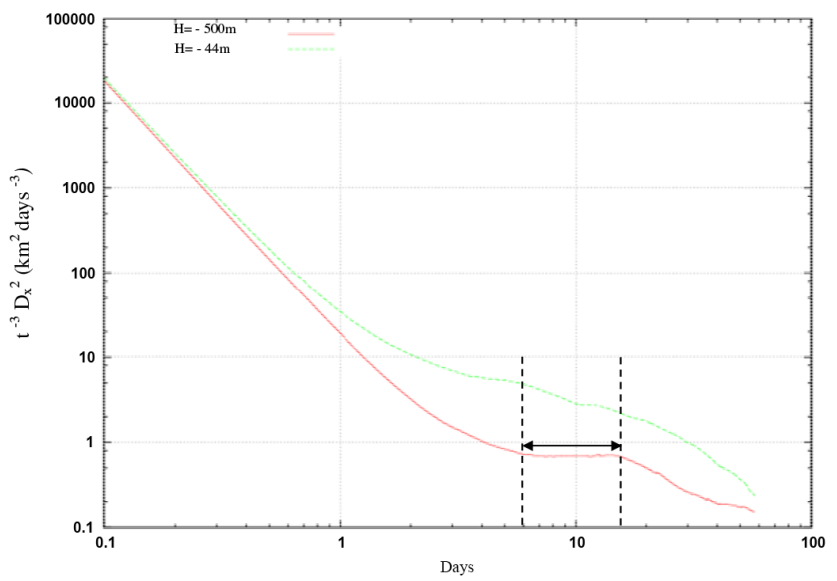

Fig. 8. The surface (green curve) and the $500 \mathrm{~m}$ depth (red curve) relative zonal dispersion divided by $t^{3}$ for $D_{0}=5.55 \mathrm{~km}$. The plateau of the $500 \mathrm{~m}$ depth extends from the sixth to the sixteenth day.

The relative diffusivity also has three phases, following those of the relative dispersion. It grows respectively as $D^{2}$ and $D^{4 / 3}$ for distances smaller or larger than roughly $20 \mathrm{~km}$.

At $500 \mathrm{~m}$ depth, the integral timescale increases and the intermediate regime becomes narrower than the surface one due to the weaker effect of vortices (which decreases with depth). The turbulence becomes more homogeneous and the Richardson law takes place.

Though the western Mediterranean Sea is a complex environment where density stratification and Earth rotation act, we observe a similarity of its coherent structures to those of two-dimensional turbulence and the presence of two inertial ranges in the energy spectrum, and the laws of relative dispersion derived in the context of two-dimensional turbulence hold well near the surface in the western Mediterranean
Basin. Indeed, the model was able to reproduce with reasonable accuracy these dispersion properties in this region.

The complementary analysis at $500 \mathrm{~m}$ depth shows that the effect of the mesoscale eddies, produced in particular by the baroclinic instability of boundary currents, is most intense at the level of their thermohaline core (that is, above $400 \mathrm{~m}$ depth), though it is known that many eddies have a dynamical influence below this core, as shown by the vertical section of horizontal velocity.

Further work will investigate the submesoscale dynamics. Indeed, the intense coherent vortices form filaments that play a significant role for marine ecosystems in the western Mediterranean Basin. The use of a biogeochemical model coupled with the physical circulation model will allow us to assess the impact of these eddies on biological processes.

Acknowledgements. The authors thank the editor of the special issue and the referees for helping them to improve this article. They are grateful to the University of Tunis El Manar-Tunisia and the University of Brest-France for support during the course of this study. This research was performed in partial fulfillment of the requirements for the $\mathrm{PhD}$ by Halima Nefzi. This work was supported by the Ministry of Higher Education, Scientific Research and Technology of Information and Communication in the frame work of the Tuniso-Fresh collaboration (DGRS/CNRS) [grant number 14R1001].

Edited by: T. Tanhua

\section{References}

Babiano, A., Basdevant, C., Leroy, P., and Sadourny, R.: Relative dispersion in two- dimensional turbulence, J. Fluid Mech., 214, 535-557, 1990.

Béranger, K., Mortier, L., and Crépon, M.: Seasonal variability of water transports through the Straits of Gibraltar, Sicily and Corsica, derived from a high resolution model of the Mediterranean circulation, Prog. Oceanogr., 66, 341-364, 2005.

Conkright, M. E., Locarnini, R. A., Garcia, H. E., O Brien, T. D., Boyer, T. P., Stephens, C., and Antonov, J. I.: World Ocean Atlas 2001: Objective Analyses, Data Statistics, and Figures, CDROM Documentation 2001, National Oceanographic Data Center, Silver Spring, MD, Report no. 17, 2002.

Da Silva, A. M., Young, C. C., and Levitus, S.: Atlas of surface marine data, algorithms and procedures. Technical Report U.S. Department of commerce, Vol. 1, NOAA, 1994.

Elhmaidi, D., Provenzale, A., and Babiano, A.: Elementary topology of the two-dimensional turbulence from a Lagrangian viewpoint and single particle dispersion, J. Fluid Mech., 257, 533558, 1993.

Elhmaidi, D., Hardenberg, J. V., and Provenzale, A.: Large-scale dissipation and filament instability in twodimensional turbulence, Phys. Rev. Lett., 95, 1-4, doi:10.1103/PhysRevLett.95.014503, 2005.

Elhmaidi, D., Nefzi, H., Carton, X., and Lili, T.: Particle dispersion in the Western Mediterranean basin, The Open Oceanography Journal, 4, 137-143, 2010. 
Haidvogel, D. B., Arango, H. G., and Hedstrom, K.: Model evaluation experiments in the north atlantic basin: simulations in nonlinear terrain-following coordinates, Dynam. Atmos. Ocean, 32, 239-281, 2000.

LaCasce, J. H. and Bower, A.: Relative dispersion in the subsurface North Atlantic, J. Mar. Res., 58, 863-894, 2000.

LaCasce, J. H. and Ohlmann, J. C.: Relative dispersion at the surface of the Gulf of Mexico, J. Mar. Res., 61, 285-312, 2003.

Lumpkin, R. and Elipot, S.: Surface drifter pair spreading in the North Atlantic, J. Geophys. Res., 115, C12017, doi:10.1029/2010JC006338, 2010.

Marchesiello, P., McWilliams, J. C., and Shchepetkin, A.: Open boundary condition for long-term integration of regional oceanic models, Ocean Model., 3, 1-21, 2001.

McWilliams, J.: The emergence of isolated coherent vortices in turbulent flow, J. Fluid Mech., 146, 21-43, 1984.

Millot, C.: Circulation in the western Mediterranean Sea, J. Mar. Syst., 20, 423-442, 1999.

Millot, C. and Taupier-Letage, I.: Additional evidence of LIW entrainment across the Algerian Basin by mesoscale eddies and not by a permanent westward flow, Prog. Oceanogr., 66, 231-250, 2005.
Ollitrault, M., Gabillet, C., and Colin De Verdière, A.: Open ocean regimes of relative dispersion, J. Fluid Mech., 533, 381-407, 2005.

Penven, P., Cambon, G., Tan, T., Marchesiello, P., and Debreu, L.: Roms_agrif/romstools user's guide - roms_agrif v2.1 - romstools v2.1, available at: http://www.romsagrif.org/index.php/ documentation (last access: 7 March 2013), 2010.

Penven, P., Marchesiello, P., Debreu, L., and Lefèvre, J.: Software tools for pre- and post processing of oceanic regional simulations, Environ. Model. Softw., 23, 660-662, 2008.

Richardson, L. F.: Atmospheric diffusion shown on a distanceneighbor graph, P. Roy. Soc. London A, 110, 709-737, 1926.

Shchepetkin, A. F. and McWilliams, J. C.: The regional oceanic modeling system (ROMS): a split-explicit, free-surface, topography following - coordinate oceanic model, Ocean Model., 9, 347-404, 2005.

Smith, W. H. F. and Sandwell, D. T.: Global sea floor topography from satellite altimetry and ship depth soundings, Science, 227, 1956-1962, 1997.

Song, Y. and Haidvogel, D. B.: A semi-implicit ocean circulation model using a generalized topography-following coordinate system, J. Comput. Phys., 115, 228-244, 1994. 